\title{
Conflicto socioambiental y rellenos sanitarios en los Pueblos de la Barranca en Zapopan, Jalisco
}

\author{
Beatriz A. Venegas Sahagún*
}

Recepción: 11 de septiembre de 2017 Aceptación: 7 de febrero de 2018

Resumen El presente artículo tiene por objetivo analizar el conflicto socioambiental que viven los habitantes de los pueblos de la Barranca, originado por el mal manejo de los rellenos sanitarios Hasar's y Picachos, en operación, y Copala y El Taray, ya clausurados, en el municipio de Zapopan, Jalisco. A causa de lo anterior, los habitantes se organizaron para defender sus derechos, su calidad de vida, sus tierras y sus ríos frente a un Estado que hace caso omiso de estas demandas. Se utilizó una metodología mixta, cuantitativa y cualitativa, la cual permitió argumentar que los conflictos socioambientales ponen en evidencia los efectos de la contaminación, que a su vez contribuyen al deterioro ambiental, el despojo y las luchas de poder existentes entre los diferentes actores.

PALABRAS CLAVE: conflicto socioambiental, rellenos sanitarios, pueblos de la Barranca, lixiviados, contaminación.

\section{Socio-environmental conflict and landfills at the "Pueblos de la Barranca" in Zapopan, Jalisco}

Abstract This paper aims to analyze the socioenvironmental conflict between the inhabitants of the "pueblos de la Barranca" caused by the poor management of the sanitary landfills, Hasar's and Picachos, in operation, and Copala and El Taray already closed, in the municipality of Zapopan, Jalisco. Because of this, the inhabitants organized themselves to defend their rights, their quality of life, their lands and their rivers, in front of a State that ignores these demands. A mixed methodology was used,

Profesora de asignatura en el Centro Universitario de Ciencias Económico Administrativas (CUCEA) de la Universidad de Guadalajara, betyvenegas@gmail.com 
quantitative and qualitative, which allowed to arguing that socioenvironmental conflicts highlight the effects of pollution, which in turn contribute to environmental deterioration, dispossession and power struggles between different actors.

KEY WORDS: socioenvironmental conflict, sanitary landfills, pueblos de la Barranca, leachate,

\section{Introducción}

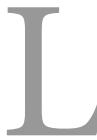

os problemas ambientales actuales presentan un gran desafío a nivel mundial especialmente en los países emergentes debido a que su población es más vulnerable que la de los países desarrollados. Dichos problemas han sido marcados como prioritarios en las diversas agendas públicas internacionales; tal fue el caso en 1992 de la Agenda 21 y actualmente de la Agenda 2030, entre otros. De acuerdo con la Organización Mundial de la Salud (OMS, s.f.), los problemas ambientales (contaminación de acuíferos, atmosférica y del suelo, asi como generación de residuos, entre otros) van en aumento, son más agudos y su afectación a la salud de la población es mayor. Esta contaminación en la mayoría de los casos ocasiona conflictos socioambientales.

Los conflictos socioambientales "implican problemas de poder de diferente escala de gestión del espacio y sus recursos ambientales; incluyen procesos de gestión en la medida en que revelan situaciones de usufructo y explotación de recursos" (Orellana, 1999, p. 334). En estos conflictos la comunidad afectada se organiza y hace frente a los actores, ya sean públicos o privados, que inciden de manera negativa en su medio ambiente.

Tal como mencionan Toledo, Garrido y Barrera Basols (2013), los conflictos socioambientales han aumentado en las últimas décadas tanto a nivel mundial como en México. Han estado latentes debido a la destrucción del medio natural, que es resultado de las políticas neoliberales (Buchanan, 2013; Toledo et al., 2013) de un Estado que tiene como prioridad expandir el capital, sin tomar en cuenta el cuidado del medio ambiente ni las repercusiones que esto puede tener.

En la literatura internacional se han señalado algunas causas de que ocurran conflictos socioambientales. Entre ellas están los impactos ambientales negativos en flora y fauna, el mal manejo de los residuos sólidos urbanos (RSU), el impacto ambiental de proyectos de desarrollo, los conflictos derivados de prácticas de gestión por parte de autoridades, el uso excesivo de los recursos renovables, la sobretensión de la capacidad de resiliencia del medio ambiente, el empobrecimiento del espacio de vida, el despojo del territorio y la incompatibilidad del uso de los recursos naturales 
(Libiszewski, 1991; Pavón, Ventura, Ribas, Serra, Saurı, \& Breton, 2002; Reboratti, 2012; Rojas \& Araujo, 2017).

En México se identificaron "los diez tipos principales de conflictos socioambientales, siendo los siguientes: agrícolas, biotecnológicos, energéticos, forestales, hidráulicos, mineros, por residuos peligrosos y rellenos sanitarios, turísticos y urbanos" (Toledo et al., 2013, p. 17). En este reconocimiento, los conflictos por rellenos sanitarios no son prioritarios, a diferencia de los causados por la minería, que han sido reconocidos como uno de los tipos de conflictos más importantes a nivel tanto nacional como internacional (Hilson, 2002; Tetreault, 2013; Buchanan, 2013; Merino, 2014).

Aunque no se reconoce entre los principales, uno de los problemas más grandes de contaminación actuales es el derivado de los RSU. Su generación, aunada a su mal manejo y disposición, ocasionan impactos al medio ambiente pues contaminan el suelo, los mantos acuíferos y el aire. Así mismo, atrae fauna nociva que altera el equilibrio del ecosistema y tiene afectaciones a la calidad de vida de la población aledaña a sitios de disposición final.

Por lo anterior, en esta investigación se trabaja el tema del mal manejo de los RSU, en concreto la mala disposición de estos, con el objetivo de analizar el conflicto socioambiental de los pueblos de Huaxtla, Milpillas y San Lorenzo -denominados pueblos de la Barranca- ocasionado por la mala disposición de los RSU, que ha traído como consecuencia la contaminación de cuerpos de agua, del suelo, y la proliferación de plagas y fauna nociva. Además de examinar el conflicto socioambiental, se presenta un breve análisis sobre el involucramiento y conocimiento de la población generadora de estos RSU, la cual vive en los municipios de Zapopan y San Pedro Tlaquepaque, respecto al conflicto socioambiental que viven las comunidades afectadas. Con esto último se pretende demostrar la existencia de un canal de comunicación respecto a estos temas.

La organización de este artículo es la siguiente: en un primer momento se discute la teoría de los conflictos socioambientales y la importancia de estos estudios, y en seguida la metodología utilizada; como un tercer punto se describe el caso de estudio, con referencia a los pueblos de la Barranca, para luego pasar a la revisión de los rellenos sanitarios y su impacto en el territorio donde se encuentran. Posteriormente se analiza la evolución de este caso, cómo pasó de ser un problema de contaminación ambiental a convertirse en un conflicto socioambiental, que incluye el involucramiento y conocimiento de la población generadora de los RSU que depositan sus residuos en los rellenos sanitarios y ocasionan este conflicto. Por último, se presentan las conclusiones derivadas de este análisis. 


\section{Los conflictos socioambientales}

En el análisis de los conflictos socioambientales resulta importante mencionar la diferencia entre este término y el de conflicto ambiental. Los primeros se manifiestan como disputas políticas, sociales, económicas, étnicas, religiosas o territoriales, o como altercados sobre recursos o intereses nacionales, o de cualquier otro tipo. Son conflictos tradicionales inducidos por una degradación ambiental (Libiszewski, 1991, p. 7), están "relacionados con el daño a los recursos naturales, donde la oposición proviene principalmente de actores exógenos, por lo común activistas de organizaciones ambientalistas" (Walter, 2009, p. 2), como es el caso que presentan Sabatini, Mena y Vergara (1996); Verduzco (2002) y Fuenzalida y Quiroz (2012), entre otros.

Los conflictos socioambientales, surgen cuando los actores endógenos sufren los efectos del daño ambiental, y por ende de los recursos naturales y la desposesión de estos, resisten y toman partido, colisionan ante los actores responsables -el Estado y actores privados - en lugar de consensuar, lo cual incrementa las tensiones entre los actores que interactúan en el territorio y generan un desencuentro o disputa (Orellana, 1999; Montenegro, 2008; Walter, 2009; Cáceres, 2015). En palabras de Tetreault y McCulligh, los conflictos socioambientales son "una confluencia de movimientos ecológicos, un nuevo frente de resistencia al desarrollo capitalista neoliberal” (2012, p. 93).

El estudio de los conflictos socioambientales tiene sus raíces en la ecología política, donde se analizan los conflictos ecológicos y distributivos, que conciernen a la naturaleza y a los seres humanos y sus relaciones sociales. Son causados por el modelo de desarrollo actual (Velázquez, Ochoa, \& Morales, 2012, p. 184; Toledo et al., 2013), basado en la producción y el consumo de bienes y servicios, así como en la explotación y el uso de los recursos naturales. Este modelo "ocasiona daños a los recursos, al patrimonio y a la salud de comunidades” (Bernache, 2012, p. 40).

De acuerdo con Leff (1993), la globalización, el desarrollo de las fuerzas productivas, las economías capitalistas y el nuevo sistema neoliberal han contribuido a una crisis socioambiental a nivel global. Esta crisis es ocasionada por la "irracionalidad económica antinatural... la cual es vinculada a los patrones de consumo de recursos y energía de los sectores opulentos de la sociedad” (p. 2). Es así como el desarrollo progresivo y las economías capitalistas han impactado en forma directa en el medio ambiente, creando una crisis socioambiental, la cual deriva en estos conflictos.

1 Entendida como "los patrones dominantes de producción y consumo, y marcando los límites del crecimiento económico". 
Los conflictos socioambientales surgen debido a la agresiva demanda de recursos como materias primas, el cambio de uso de suelo, la introducción de semillas genéticamente modificadas, la privatización y el control del agua y la tierra por compañías transnacionales; a los residuos sólidos, la desposesión, etc. (Martín, 2007; Cáceres, 2015; Tetreault et al., 2012b), prácticas y eventos que repercuten no solo en el medio ambiente sino también en la salud y la calidad de vida de la población afectada.

Dado el hartazgo de las comunidades por la indiferencia del Estado ante estos problemas y la falta de confianza en él, que es una variable importante para el desarrollo de los conflictos ambientales (Espluga, Paredes, Gamer, \& Sola, 2008), ${ }^{2}$ muchos grupos han optado por organizarse y formar alianzas y redes con diversos actores a fin de enfrentar el poder; el cual en muchos casos es la mancuerna del Estado con actores privados (Espluga et al., 2008; Tetreault et al., 2012). Estos últimos hacen demandas y sugerencias que sobrepasan las libertades, el poder de decisión o la participación en la gestión de las comunidades afectadas.

De acuerdo con Tetreault et al. (2012) y Toledo et al. (2013), los conflictos socioambientales han ido en aumento en México en las últimas dos décadas. Sin embargo, no solo ocurren en este país, sino también en otros países emergentes de América Latina (Martín, 2007, p. 24; Merino, 2014), e incluso se llegan a tener en países desarrollados (por ejemplo la oposición al fraking en varios países europeos o la lucha contra la pypeline en Dakota, Estados Unidos).

Como ya es sabido, e incluso se ha mencionado en reiteradas ocasiones en las agendas internacionales, la población rural y de escasos recursos es la más vulnerable ante la contaminación ambiental, el despojo de tierras, la privatización, etc. El causante principal de estos males es el Estado, por su política capitalista neoliberal. Tal como lo menciona Harvey (2003), el Estado, en su afán de incrementar su capital económico, ha trasferido bienes públicos a sectores privados, que tratan los recursos como si fueran infinitos, con lo cual destruyen y afectan la dinámica de los ecosistemas, y esto tiene repercusiones directas en el ser humano. Es así como se llega a la acumulación por desposesión.

Lo dicho hasta aquí esclarece la relación de los conflictos socioambientales con el ecologismo de los pobres. Este último fue estudiado por Martínez (1994, p. 20), quien menciona que "la historia está llena de movimientos ecologistas de los pobres, es decir, de conflictos sociales con contenido ecológico y cuyos actores tenían una percepción ecológica". Este tipo de ecologismo alude a la importancia del acceso a los bienes comunes por parte de las comunidades rurales, los cuales son su medio de subsistencia.

"El concepto de 'confianza' en el centro de las explicaciones sobre conflictos socioambientales" (Espluga et al., 2008, p. 255). 
En relación con los conceptos presentados, aún es necesario identificar cuándo se desarrolla un conflicto socioambiental. De acuerdo con Quintana (2008, p. 12), los conflictos socioambientales constan de seis etapas: cooperación, indiferencia, competencia, tensión en aumento, conflicto como tal y crisis. Por eso para alcanzar el objetivo de este artículo se identifican tales etapas y se pretende contestar a lo largo del texto las siguientes preguntas, propuestas por Tetreault et al. (2012): “Cuáles son las causas subyacentes de estos conflictos? ¿Quiénes son los protagonistas? ¿Cuáles son sus demandas, propuestas y estrategias? ¿Cómo interactúan con los actores externos? ¿En qué medida han contribuido a proteger o sanear el medio ambiente, patrimonio de todos?" ( p. 14). A estas preguntas se agrega la siguiente: ¿existe involucramiento de los actores que por sus externalidades son parte generadora del conflicto socioambiental? Esta última con el fin de analizar los conflictos socioambientales no solo entre los actores afectados y el Estado o actores privados, sino agregando a la arena la población que, por sus actividades y el modelo de desarrollo actual, generan impactos directos o indirectos en el medio ambiente.

\section{Estrategia metodológica}

Esta investigación tiene como objetivo analizar el conflicto socioambiental que vive la población de los pueblos de la Barranca. Para poder alcanzarlo se utilizó una estrategia metodológica mixta, de carácter cualitativo y cuantitativo, la cual se sustentó en actividades de trabajo empírico y documental.

En cuanto al trabajo cualitativo, el conocimiento generado para este estudio se derivó del trabajo de campo, que se llevó cabo en dos fases. La primera comprendió del primero de mayo al 31 agosto de 2015; la segunda, del primero de enero al 31 de agosto de 2016. En la investigación de campo se realizaron las siguientes actividades: 1) recorridos de campo en las comunidades afectadas; 2) visitas en los perímetros de los sitios de disposición final Hasar's y Picachos; 3) entrevistas estructuradas a actores clave de diferentes dependencias e instituciones, tales como el director de la Procuraduría Estatal de Protección al Ambiente (PROEPA), los directores de medio ambiente de los municipios de Zapopan y San Pedro Tlaquepaque, funcionarios de la Secretaría de Medio Ambiente y Desarrollo Territorial del Estado de Jalisco (Semadet), un profesor investigador del Centro de Investigaciones y Estudios Superiores en Antropología Social (CIESAS); 4) entrevistas no estructuradas a la población de las comunidades de Huaxtla, Milpillas y San Lorenzo, así como a los líderes de estas comunidades; 5) revisión de periódicos e información solicitada a los Ayuntamientos de Zapopan y San Pedro Tlaquepaque, la Semadet y la PROEPA, a través del portal de Transparencia del Estado de Jalisco, el sistema Infomex Jalisco. 
Con el trabajo cuantitativo se pretendió analizar el conocimiento que la población generadora de los RSU tiene sobre el conflicto socioambiental que enfrentan los pueblos de la Barranca. Este ejercicio se basó en encuestas dirigidas a usuarios/generadores del servicio de recolección de los municipios de Zapopan y San Pedro Tlaquepaque. Se aplicaron 385 cuestionarios entre los habitantes de ambos municipios; el número de ellos fue obtenido de acuerdo con la fórmula del tamaño de una muestra para población finita. Para calcularlo se eligió a la población de entre 18 y 69 años de edad que es usuaria del servicio de recolección, con un nivel de confianza del 95 \% y un margen de error del $5 \%$. Se llevó a cabo un muestreo estratificado por zonas de marginación (alta, media y baja), se aplicaron 123 cuestionarios en San Pedro Tlaquepaque y 262 en Zapopan. El número de cuestionarios aplicados se basó en la cantidad de habitantes de cada uno, para que la muestra fuera representativa. Las preguntas del cuestionario responden a los siguientes tipos de información: conocimiento, opiniones, actitudes, comportamiento y atributos. Fueron preguntas cerradas, que varían pues se conjugan las de múltiples respuestas (considerando la escala de Likert en algunos casos) con las de dos opciones, de escala de calificación y con la opción de otros. ${ }^{3}$

\section{Los pueblos de la Barranca:}

la importancia de los ríos para sus actividades agrícolas

Las comunidades de Huaxtla, Milpillas y San Lorenzo pertenecen al municipio de Zapopan, Jalisco. Se encuentran en la ruta de Zapopan a San Cristóbal de la Barranca, a escasos veinte kilómetros de la mancha urbana de la zona metropolitana de Guadalajara (ZMG), dentro del ecosistema de la Barranca de Huentitán o Barranca del río Santiago.

La Barranca del río Santiago "es el accidente geográfico más importante del occidente del país debido a su gran biodiversidad” (Escobar \& Macías, 2008); de acuerdo con Jalomo, esta biodiversidad "hace que tenga un alto nivel de fragilidad como ecosistema" (2013, p. 272), a lo que se suma el hecho de la cercanía de una de las zonas metropolitanas más grandes de nuestro país. En 2004, el Congreso del Estado de

\footnotetext{
El cuestionario se diseñó con 38 preguntas, agrupadas bajo los siguientes temas:

Cantidad y tipo de residuos generados en los hogares, opinión sobre el sistema de recolección, limpieza y responsabilidad de los actores en relación con los residuos, infraestructura para la gestión de los residuos, cultura ambiental en materia de residuos, conocimiento sobre la contaminación, disposición final e impacto ambiental de los residuos.

Para este artículo se procesaron los datos solo del tema "Conocimiento sobre la contaminación, disposición final e impacto ambiental de los residuos".
} 
Jalisco decretó la zona de la Barranca comprendida en el municipio de Zapopan como área natural protegida bajo la categoría de Área Municipal de Protección Hidrológica. ${ }^{4}$ Con esto se determinó una superficie de 17,729.91 hectáreas que colinda con los municipios de Guadalajara, San Cristóbal de la Barranca, Ixtlahuacán del Río, Amatitán, Tequila y la ZMG.

De acuerdo con el Instituto Nacional de Estadística y Geografía (INEGI) (2010), estas comunidades tienen un total de 170 habitantes distribuidos en 35 familias, la mayoría son adultos y adultos mayores. Antes la población era mayor, pero a causa de la contaminación los habitantes han emigrado a la zona urbana o a Estados Unidos (entrevista con un habitante de Huaxtla, 16 de marzo de 2004). Estas comunidades han sido consideradas como del ámbito rural y con un grado de marginación alto (INEGI, 2010).

Las principales actividades económicas que se realizan en esta zona son la agricultura y la ganadería. De acuerdo con Casillas (s.f.) la producción agrícola de los pueblos de la Barranca se basa en prácticas orgánicas y semiorgánicas, entre las que se encuentra la producción de mango y maíz, así como pequeños huertos de leguminosas y verduras para consumo propio. En cuanto a la ganadería, incluyen vacas de engorda y lecheras, así como caballos. Otra actividad económica es el turismo, con un menor impacto, ya que existe un balneario en Huaxtla.

Si bien, estas comunidades se localizan en un ecosistema rico en biodiversidad que permite las actividades agrícolas y ganaderas, también son vecinos de grandes rellenos sanitarios: Picachos y Hasar's (aún en operación) y Copala y El Taray (cerrados), que se han convertido en grandes focos de contaminación (véase la figura 1).

Antes de que las comunidades tuvieran problemas de contaminación por lixiviados ${ }^{5}$ ocasionados por los rellenos sanitarios ya señalados, contaban con un balneario en Milpillas que era abastecido con aguas del Arroyo Grande Milpillas. Llevaban a cabo, además, actividades de pesca para su consumo. Este arroyo era una de las principales fuentes de abastecimiento de agua de la comunidad y de recreación junto con el río Santiago. Actualmente ambos están contaminados; el primero desemboca en el segundo, con lo que aumentan los niveles de contaminación del río Santiago.

4 El “Área Municipal de Protección Hidrológica es aquella que se destina a la preservación de ríos, manantiales y aguas subterráneas, a través de la protección de cuencas, áreas boscosas, llanuras y todas aquellas áreas que tengan impacto en las fuentes de producción y/o abastecimientos de agua" (Ayuntamiento de Zapopan, 2012, p. 11).

5 Lixiviado es el "Líquido que se forma por la reacción, arrastre o filtrado de los materiales que constituyen los residuos y que contiene en forma disuelta o en suspensión, sustancias que pueden infiltrarse en los suelos o escurrirse fuera de los sitios en los que se depositan los residuos" (DOF, 2003,p.5). 

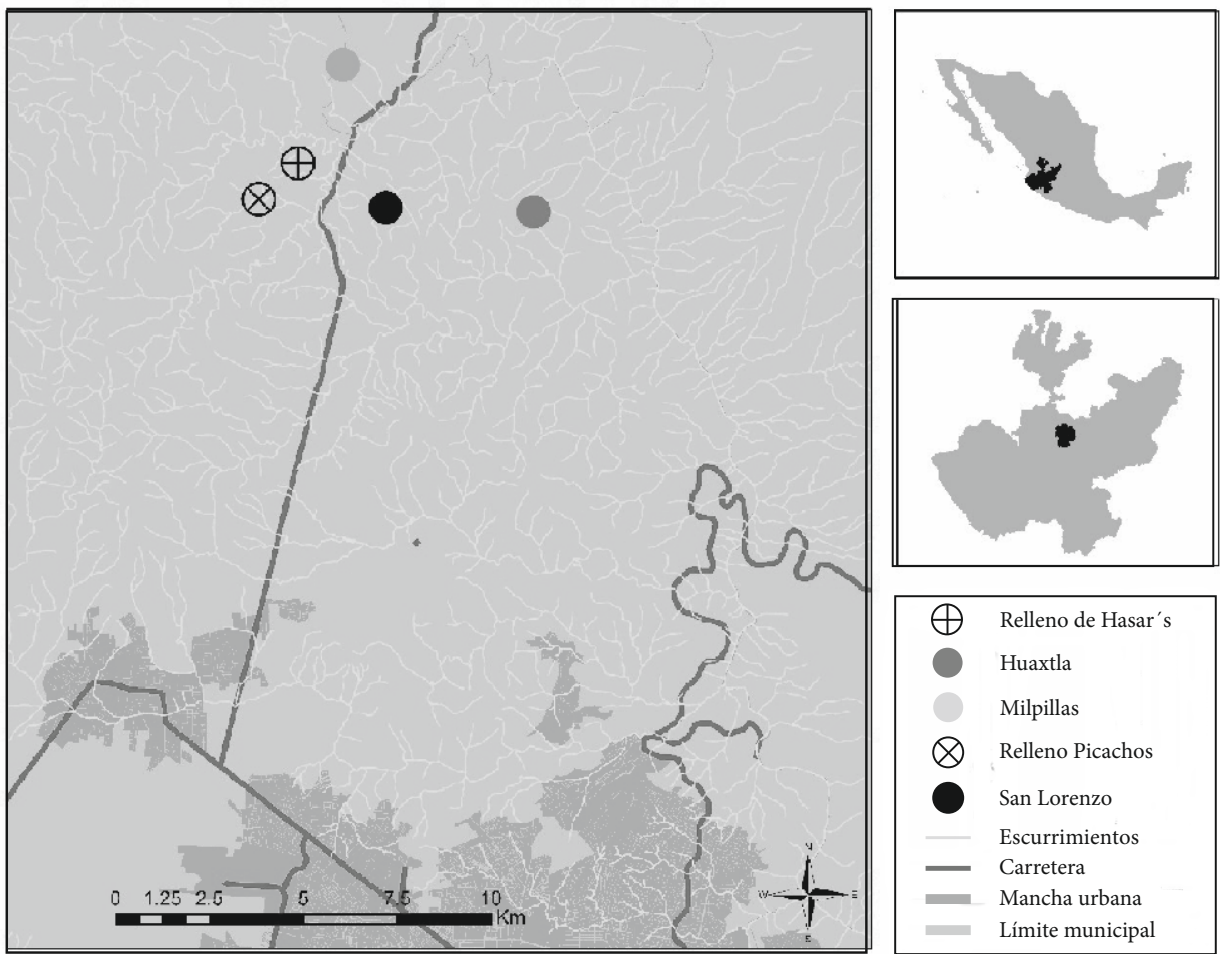

$\bigoplus$ Relleno de Hasar's

Huaxtla

Milpillas

$\bigotimes$ Relleno Picachos

San Lorenzo

Escurrimientos

Carretera

Mancha urbana

Límite municipal

Fuente: Elaboración propia con datos del INEGI (2010b).

Hoy en día las comunidades se abastecen de ojos de agua, que van en detrimento. A los habitantes de Milpillas se les suministra por medio de pipas para el uso diario; esta es una de las causas por las cuales las personas han emigrado.

\section{Los rellenos sanitarios}

Los residuos que generan las personas han sido un problema desde tiempos antiguos. Su disposición ha variado a lo largo de la historia. Medina (1999, pp. 8-9) menciona tres métodos utilizados por antiguas civilizaciones, los cuales dieron paso a que hoy en día se utilicen rellenos sanitarios. ${ }^{6}$ 
En México se emplea actualmente el método de depositar los RSU y el manejo especial en forma definitiva en los sitios de disposición final, los cuales se clasifican de diferentes maneras de acuerdo con la cantidad de toneladas que reciben por día (véase el cuadro 1).

CUADRO 1. Categorías de los sitios de disposición final

\begin{tabular}{cl} 
Tipo & Tonelaje recibido (toneladas al día) \\
\hline A & Mayor de 100 \\
B & de 50 a 100 \\
C & Más de 10 y menos de 50 \\
D & Menos de 10
\end{tabular}

Fuente: NOM-083-SEMARNAT-2003.

De acuerdo con el INEGI, hasta 2010 en todo México había 1,881 sitios de disposición final, de los cuales tan solo 230 eran rellenos sanitarios y 1,643 tiraderos a cielo abierto. En Jalisco se cuenta con 108 sitios de disposición final; de estos, 32 son rellenos sanitarios y 76 tiraderos a cielo abierto. Estos últimos no cumplen con lo que establece la NOM-083-SEMARNAT-2003.

En el municipio de Zapopan hay dos sitios de disposición final cerrados: Copala y El Taray, y dos en operación: Hasar's y Picachos. El vertedero de Copala se encuentra en el kilómetro 15 de la carretera de Zapopan a San Cristóbal de la Barranca; operó en 1994 y 1995 y se localiza a escasos 700 metros de asentamientos humanos. El lixiviado que se genera en este sitio "fluye en pequeños escurrimientos que brotan en la base de los taludes del entierro y se acumula poco a poco formando pequeños arroyos que corren pendiente abajo hasta dos lagunas de oxidación [...] durante la época de lluvia estas lagunas se desbordan y los lixiviados van directamente al cauce del arroyo lateral y fluyen corriente abajo" (Bernache, 2011, p. 412).

El Taray está a tan solo dos kilómetros del vertedero de Copala, en el kilómetro 17 de la misma carretera y operó de 1995 a 2001. El control de los lixiviados es limitado y terminan en los cauces del arroyo (Bernache, 2011, p. 419). Estos dos sitios se encuentran en el lado opuesto de Picachos y Hasar's.

ocasionaba que con el paso de las personas se aplanara y subiera el nivel de la calle. El segundo se utilizó en Creta del año 3000 al 1000 a.C. y consistió en transportar los residuos fuera de la ciudad y depositarlos en un hoyo, que era cubierto de tierra, lo cual dio paso a los rellenos actuales. El tercero era recuperar, reutilizar y reciclar los residuos; un ejemplo de esto es lo que ocurría en el México prehispánico del siglo XVI: las heces se recuperaban y reutilizaban en las chinampas (Medina, 1999, pp. 8-9). 
Los rellenos sanitarios de Hasar's y Picachos también se encuentran por la carretera a Colotlán, en el kilómetro 17.5; debido a que sus terrenos colindan, el foco de contaminación y de afectación aumenta. De acuerdo con Gran Castro (2016, p. 97), el relleno sanitario de Hasar's "inició desde 1998 a manera de relleno sanitario de tipo privado, administrado primeramente por la compañía Recolectora de Occidente S.A. y posteriormente por grupo GEN. En un principio captaba pocos residuos de la ciudad, pero, a partir de 2001, tras comenzar a trabajar también como planta de transferencia, la cantidad de residuos que captaba aumentó drásticamente". En la actualidad recibe residuos tanto privados como del municipio de San Pedro Tlaquepaque.

Picachos inició sus operaciones en 2001; fue creado con el propósito de ser un relleno metropolitano. Está asentado sobre el cauce del arroyo el Pedregal, tributario del Arroyo Grande Milpillas. El Pedregal fue entubado y sobre él se depositaron grandes cantidades de residuos sólidos. Este relleno es actualmente exclusivo del municipio de Zapopan. De acuerdo con Gran Castro (2016, p. 100), "se estima que, entre ambos sitios, ingresan 2,500 toneladas diariamente".

\section{Antecedentes del conflicto socioambiental ocasionado por los rellenos sanitarios}

Tanto los sitios de disposición final clausurados como los que están en operación han sido objeto de escrutinio y demandas. Los primeros tuvieron denuncias ante la Comisión Estatal de Derechos Humanos de Jalisco (CEDHJ), los segundos, además de ser objeto de denuncias, fueron multados por la PROEPA.

En 2008, la CEDHJ recibió una denuncia de la población aledaña a los rellenos sanitarios, que en seguimiento a las recomendaciones con el número 2/99, "advirtió el poco interés de la Procuraduría General de Justicia del Estado por investigar los posibles delitos en materia ambiental en los que incurrieron los servidores públicos de los ayuntamientos de la ZMG por la falta de una captación y tratamiento de los lixiviados en los vertederos ya clausurados" (CEDHJ, 2008, p. 193). Esto debido a que los vertederos no siguieron el procedimiento establecido en la NOM-083-SEMARNAT2003.

En 2009, de acuerdo con Bernache, el túnel que pasaba por debajo de Picachos se fracturó y permitió la filtración de lixiviados (Proceso, 2010) sobre el arroyo el Pedregal. En el mismo año colapsó y contaminó este arroyo. Esto derivó en que en 2010 tuviera dos observaciones respecto al manejo de lixiviados; la PROEPA señaló que necesitaba un "sistema que garantice la captación y extracción de lixiviados, acciones necesarias para el control de lixiviados en los canales de la celda 1 y 2 así como en las fosas 2 y 3 provenientes del lado sur y el canal de agua pluvial" (Proceso, 2010). 
En 2011, la CEDHJ solicitó al Ayuntamiento de Zapopan "Construir en el relleno sanitario de Picachos un adecuado sistema que garantice la captación y extracción de lixiviados, elaborado con materiales de calidad y de alta densidad para evitar a toda costa el derramamiento de los referidos líquidos" (CEDHJ, 2011, p. 32). Esto como respuesta a la queja que presentaron las comunidades barranqueñas por la contaminación con lixiviados en su zona.

Dos años después, en 2013, se le hizo la observación siguiente al Ayuntamiento de Zapopan: "controlar la dispersión y compactación de residuos, fauna nociva, no contar con programa que incluya la medición y control de impactos ambientales, así como un programa de monitoreo, drenar la fosa que se ubica en los puntos 13Q0660385 y 2213730, sanear los puntos impactados por descargas de lixiviados, drenar y sanear el predio conocido como El Tajo, obtener la autorización en materia de impacto ambiental para la instalación de rellenos sanitarios" (CEDHJ, 2011, p. 32). Esta observación no procedió. ${ }^{7}$

Al sitio de disposición final Hasar's se le han aplicado multas por lixiviados e incendios, y ha ganado la mayoría de los casos en los que ha sido denunciado (PROEPA, 2016).

Los sitios de disposición final mencionados se encuentran dentro de un mismo polígono, el cual presenta contaminación por lixiviados, están a 0.47 kilómetros de un río de corriente temporal y a un kilómetro de un cuerpo de agua. La contaminación que generan los cuatro sitios de disposición final ha sido expuesta por los afectados. Sin embargo, no hubo soluciones, por lo que inició el conflicto socioambiental con las comunidades de Huaxtla, Milpillas y San Lorenzo.

\section{El paso de problema de contaminación ambiental a conflicto socioambiental}

¡Fuera basurales asesinos! Ni ambientalistas, ni ecologistas, somos pueblos en resistencia. ${ }^{8}$

Los problemas socioambientales en las comunidades de Huaxtla, Milpillas y San Lorenzo, del municipio de Zapopan, Jalisco, surgieron a partir de la contaminación del

8 Véase http://pueblosbarrancasantiago.blogspot.mx/2014/

7 De acuerdo con la NOM-083-SEMARNAT-2003 Restricción por afectación a obras civiles o áreas naturales protegidas, los rellenos sanitarios no se deben ubicar en sitios de áreas naturales protegidas, deben localizarse a una distancia mínima de 1,500 metros del límite de la traza urbana, así como de poblaciones rurales de hasta 2,500 habitantes. Así mismo, la distancia de ubicación del sitio con respecto a cuerpos de agua superficiales con caudal continuo debe ser de 1,000 metros como mínimo y contar con una zona de amortiguamiento tal que pueda retener el caudal de la precipitación pluvial máxima presentada en los últimos diez años en la cuenca, definida por los canales perimetrales de la zona. 
recurso hídrico, en especial por la contaminación del Arroyo Grande Milpillas, ocasionada por el derrame de lixiviados de los sitios de disposición final ya mencionados, lo cual ocasionó afectaciones a la salud de la población, su calidad de vida y al ecosistema de las localidades. El caso fue presentado a la CDEHJ y la Asamblea Nacional de Afectados Ambientales (ANAA). Hubo demandas ante la PROEPA, se acudió al apoyo y se hizo trabajo en conjunto con la asociación Un Salto de Vida (USV), los Geocomunes e instituciones de educación como el Centro de Investigaciones y Estudios Superiores en Antropología Social (CIESAS-Occidente), el Instituto Tecnológico y de Estudios Superiores de Occidente (ITESO) y con alumnos de la Universidad de Guadalajara.

Es importante mencionar el contexto en que los sitios de disposición final llegaron a ese lugar. Esta historia la explica un habitante de Huaxtla:

Nuestros abuelos dieron permiso para que se instalara el primer sitio de disposición, ellos no lo vieron como una amenaza al medio ambiente debido a que antes tiraban doscientas toneladas de basura diarias y ahorita tiran casi tres mil; ellos no hubieran aceptado si les hubieran dicho que se iban a morir, que sus nietos se iban a morir con la contaminación. No hubieran aceptado nunca (entrevista efectuada el 16 de marzo de 2004).

Un habitante de San Lorenzo comentó:

El basurero fue un engaño que le hicieron a la gente porque les trajeron una maqueta, porque les dijeron que todo iba a ser bien bonito, que hasta iban a hacer un parque ahí. Una maqueta así bien bonita, bien arbolado, que iba a haber empleo para la gente. Ya traían un rollo bien organizado [haciendo referencia al gobierno estatal y municipal], pero nunca le dijeron a la gente que iba a haber la contaminación que hay (entrevista efectuada el 16 de marzo de 2004).

Fue así como se instaló el primer sitio de disposición final, el de Copala. Esto abrió el camino para que se establecieran los demás sitios. La afectación por lixiviados no estaba presente aún, lo que reafirma la teoría de los conflictos socioambientales, en que las más afectadas y vulnerables resultan ser las comunidades rurales, que defienden los bienes comunes.

Al paso de los años, con el cierre y la apertura de los sitios mencionados se escaparon los lixiviados y se presentaron primero en el arroyo el Pedregal, y de allí pasaron al Arroyo Grande Milpillas, lo cual ocasionó el cierre del balneario del mismo nombre en 2009. Actualmente el balneario está destruido y el agua del arroyo no es apta para el consumo humano. Este arroyo pasa por diversos predios, donde aún hay animales que beben sus aguas; los pobladores tratan de comprar agua en pipas para evitar que su ganado ingiera este líquido, pero la situación es insostenible económicamente y la ganadería ha resultado afectada. 
La agricultura también ha tenido sus pérdidas, los cultivos han sido atacados por nuevas plagas, tal como lo remarcan los habitantes de la zona, por lo que se ha gastado en plaguicidas. Se han reducido los huertos de autoconsumo debido a que no cuentan con el recurso hídrico para el riego y deben ir a la zona urbana a abastecerse de frutas y verduras. Otros problemas que se han detectado en la zona son los relacionados con la salud de la población; los habitantes mencionan que se presentan más enfermedades gastrointestinales, y en algunos casos infecciones en la piel. Así mismo, no pueden ingerir agua de este arroyo; sus fuentes de abastecimiento se están terminando.

De acuerdo con un habitante de Huaxtla, lo anterior ocasionó que diversas familias se fueran a vivir a otros lugares, pues la situación se volvió insostenible. Un ejemplo de las afectaciones es la cascada de Huaxtla, que fue un lugar de esparcimiento y turismo y actualmente está contaminada con lixiviados.

El año 2009 fue un parteaguas para las comunidades de la Barranca, "los derrames de lixiviados al Arroyo Grande de Milpillas fueron constantes" (Bernache, 2012, p. 47), y coincidieron con la fragmentación del túnel por el que pasa el arroyo el Pedregal bajo Picachos. Los habitantes hicieron demandas al Ayuntamiento de Zapopan, pero estas no fueron atendidas, por lo que decidieron cerrar el acceso a Picachos y Hasar's, que comparten el camino de entrada: "150 pobladores afectados por los basureros Picachos y Hasar's, cerraron el acceso a ambos sitios, en espera de una mesa de diálogo con las autoridades competentes [...] El Alcalde de Zapopan, Sánchez Aldana, ni siquiera quiso reconocer que hay escurrimiento de lixiviados" (Vargas, 2009).

En el mismo año se realizaron mesas de trabajo y de diálogo, con lo que inició la etapa de cooperación, que tuvo como resultado la firma de un acuerdo y la formación de una comisión interinstitucional "con representantes de las tres partes involucradas, y con académicos de la Universidad de Guadalajara, otros centros de investigación reconocidos, la Comisión Estatal del Agua, la Procuraduría Estatal de Protección al Ambiente y la Secretaría de Medio Ambiente para el Desarrollo Sustentable del Estado de Jalisco" (Bernache, 2012, pp. 47-48). En el acuerdo, el presidente municipal, Juan Sánchez Aldana, se comprometió a:

...no permitir que se derramara una gota de lixiviados al río, que iban a hacer estudios geológicos o hidrológicos para ver si no había infiltraciones, que iban a sanear la cuenca, y pues de eso no hicieron nada. Ni hicieron estudios, ni sanearon la cuenca, ni evitaron que se derramaran los lixiviados (entrevista con habitante de Huaxtla, 16 de marzo de 2004). ${ }^{9}$

Además de las mesas de trabajo, el gobierno realizó un análisis de los lixiviados que emergen de los sitios de disposición final, mientras que los habitantes de las

$9 \quad$ En este apartado se presenta la etapa de indiferencia por parte del Estado. 
comunidades hicieron otro por su cuenta. Los resultados del gobierno y los obtenidos por las comunidades fueron diferentes aunque las muestras fueron tomadas el mismo día, a la misma hora y en los mismos puntos. El Ayuntamiento de Zapopan argumentaba que los lixiviados de Hasar's y Picachos no contenían carga contaminante alguna, mientras que los resultados obtenidos por las comunidades mostraron "niveles de cianuros que rebasan los parámetros de las normas oficiales, la presencia de cromo en niveles altos que también superan la normatividad, la demanda química y bioquímica de oxígeno, nitratos, $\mathrm{pH}$, sólidos suspendidos y otros más que también rebasaban los niveles que marcan las normas de descargas de aguas en México" (Bernache, 2012, p. 48). ${ }^{10}$

Los resultados de los análisis no bastaron para poner fin al desastre ecológico, los habitantes de las comunidades seguían dando pruebas de las afectaciones por lixiviados. En 2010, los habitantes de las comunidades, ya integrados a la Asamblea Regional de Afectados Ambientales, realizaron otro cierre del ingreso a los rellenos sanitarios en protesta por el incumplimiento del acuerdo de 2009 por parte del gobierno municipal (Huérfano, 2010), lo que dio inició a la etapa de tensiones, y se dio seguimiento a la propuesta de Quintana (2008).

Al no obtener respuestas del gobierno (el Ayuntamiento de Zapopan, la PROEPA, la Semades ${ }^{11}$ ) ni del sector privado (Hasar's), los afectados expusieron su caso ante la CEDHJ. En la queja 11827/11/I presentada ante la CEDHJ se expresan diversas recomendaciones para minimizar el impacto de los lixiviados y subsanar el daño ambiental en la zona. Estas recomendaciones fueron dirigidas tanto al Ayuntamiento de Zapopan, a cargo de Picachos, como a la empresa Hasar's yla Semades.

En 2012, año de cambio de gobierno municipal en Zapopan, los habitantes de las comunidades realizaron otro bloqueo debido al incumplimiento de las acciones establecidas en el convenio (Por un Salto Digno, 2012). A sus demandas por mejorar su calidad de vida y el saneamiento del río se unieron actores de la organización Un Salto de Vida, y recibieron el apoyo de Vías Verdes A.C. y de académicos de la Universidad de Guadalajara, el ITESO y el CIESAS, así como de la ANAA. Para Quintana (2008), este era ya un conflicto.

Además de hacer este bloqueo, las comunidades presentaron ese mismo año ante la CEDHJ la queja 180/12/I, donde se señala la violación a su derecho a la salud y a un ambiente sano. Entre las recomendaciones que hace la CEDHJ figuran el monitoreo de lixiviados y realizar análisis de los mantos acuíferos contiguos a Picachos, Copala y El

10 En este punto inició la etapa de competencia con la realización de análisis a la par para comprobar los daños ambientales.

11 En 2012 se cambió el nombre de la Secretaría de Medio Ambiente para el Desarrollo Sustentable (Semades) por el de Secretaría de Medio Ambiente y Desarrollo Territorial (Semadet). 
Taray; así mismo, se les pide al Ayuntamiento de Zapopan y a la Semades que cumplan con lo establecido tanto en la ley como en el convenio celebrado con las comunidades.

En 2014, las comunidades volvieron a tomar el camino rumbo a los sitios de disposición final e impidieron el paso de los camiones recolectores. Ante esta situación, de acuerdo con un habitante de Milpillas, el Ayuntamiento de Zapopan envió a más de 200 pepenadores de Picachos a que movieran los carros y camiones que bloqueaban el acceso.

El último cerrón que les hicimos, como ya vieron que no podían con nosotros, pues nos echaron a los pepenadores [...] nos sacaron en peso, nos movieron las camionetas, nos hicieron como quisieron (entrevista con habitante de Milpillas, 16 de marzo de 2004).

Las demandas por una mejor calidad de vida y el saneamiento de los cuerpos de agua continúan por parte de las autodenominadas comunidades en resistencia. Los habitantes de las comunidades realizaron ante diversas instancias demandas y propuestas de solución a los responsables de ellas. Esto responde una de las preguntas de Tetreault et al. (2013). Las propuestas y estrategias que presentaron el $18 \mathrm{de}$ abril de $2014^{12}$ giraron en torno a los siguientes temas:

1. A los gobiernos municipal, estatal y federal se les propuso el control y tratamiento de los lixiviados de acuerdo con la NOM-083-SEMARNAT-2008. Esto incluye geomembranas adecuadas en diversas celdas y el funcionamiento correcto de plantas de tratamiento. Así mismo, se plantearon la capacitación del personal que labora en el relleno, mejores condiciones de trabajo para los pepenadores y el pago de los daños ocasionados a los habitantes a causa de las descargas de lixiviados.

2. Para la empresa Hasar's se propuso que dejara de arrojar lixiviados a la zona norte, sobre el arroyo de Mesa de San Juan, brazo del Milpillas; que suspendiera la quema de basura y la irrigación de los cerros de basura con lixiviados, así como que respondiera por los daños pasados y futuros a los pueblos y el patrimonio cultural y natural de la Barranca del río Santiago.

Estas propuestas muestran el interés de los grupos por resolver este problema en forma rápida y precisa.

Un punto importante que no se analiza con frecuencia en los conflictos socioambientales es el involucramiento de la población general, que por sus hábitos y prácticas de consumo genera externalidades, en este caso RSU que van a parar a los sitios de disposición final. Por ello en este punto se intenta responder a la pregunta: ¿existe involucramiento por parte de la población que produce externalidades que son parte generadora del conflicto socioambiental? En este caso se refiere concretamente a la población Zapopan y San Pedro Tlaquepaque, ya que sus RSU son depositados en los

\footnotetext{
12 Véasehttp://pueblosbarrancasantiago.blogspot.mx/
} 
sitios de disposición final mencionados.

Para responder a la pregunta anterior se aplicó un cuestionario que aborda esta problemática. Los resultados se presentan en el cuadro 2.

CUADRO 2. Porcentajes de conocimiento de los habitantes de Zapopan y San Pedro Tlaquepaque sobre el sitio de disposición final

\begin{tabular}{lcc}
\multicolumn{1}{c}{ Pregunta } & Sí & No \\
\hline $\begin{array}{l}\text { ¿Sabe usted dónde, en qué sitio, se deposita la basura de su municipio? } \\
\text { ¿Sabe a dónde va la basura, qué se hace con la basura después de }\end{array}$ & $27 \%$ & $73 \%$ \\
que se recolecta? & $29 \%$ & $71 \%$ \\
¿Conoce cómo funcionan los sitios de disposición final? & $16 \%$ & $84 \%$ \\
$\begin{array}{l}\text { ¿Conoce o ha escuchado alguna noticia sobre problemas de } \\
\text { contaminación ocasionados por la basura en la ciudad? }\end{array}$ & $56 \%$ \\
\hline
\end{tabular}

Fuente: Elaboración propia con datos obtenidos del cuestionario "La gestión de los residuos sólidos urbanos. ¿Un modelo de gobernanza sustentable para la sociedad? El caso de dos municipios de la zona metropolitana de Guadalajara", aplicado en noviembre y diciembre de 2015.

El cuadro anterior muestra que la mayoría de la población no tiene conocimiento sobre el destino de los residuos sólidos que genera ni del funcionamiento de los sitios de disposición final. La población no se involucra en los procesos de lucha para mejorar la gestión de los RSU, que incluye un sitio de disposición final controlado, que cumpla con la NOM-083-SEMARNAT-2003 y no tenga impactos negativos en el medio ambiente.

En cuanto a las noticias sobre los problemas relacionados con los residuos, evidencian la falta de comunicación acerca de este tema, pues la mayor parte de ellas giran en torno al bloqueo del paso del agua en las alcantarillas, lo cual provoca inundaciones en la ciudad e impacta directamente en las actividades cotidianas. Las noticias relacionadas con el derrame de lixiviados y sobre los sitios de disposición final las llegó a conocer el $1 \%$ de la población encuestada, por lo que se da más difusión a las noticias relacionadas con residuos que afectan directamente a la zona urbana.

Además de las preguntas anteriores, se preguntó a la población sobre su conocimiento acerca del lugar y la forma de disposición de los RSU. El 45 \% intuye que al enterrar la basura en un sitio (sin conocer sus especificaciones) genera o puede generar contaminación; el $38 \%$ argumenta que causa mucha contaminación; el $13 \%$ opina que se queda ya segura y no causa ningún problema al medio ambiente, y el $4 \%$ no sabe qué pasa en estos sitios.

Lo anterior supone que existe una población receptiva; sin embargo, la poca comunicación e información que se genera sobre los rellenos sanitarios y sus afecta- 
ciones es causa de esta desinformación.

Los resultados expuestos anteriormente se sintetizan enseguida de acuerdo con las etapas de los conflictos socioambientales propuestas por Quintana (2008, p. 12):

1. Cooperación. Los habitantes de las comunidades de Huaxtla, Milpillas y San Lorenzo se dirigieron en primera instancia a los gobiernos municipal y estatal. Les plantearon mesas de diálogo, de las que surgió el convenio de trabajo y la comisión interinstitucional para vigilar su cumplimiento.

2. Indiferencia. Esta se dio por parte del Estado y de la empresa privada Hasar's, pues incumplieron el convenio y no presentaron soluciones. Las presentaron los habitantes de la Barranca, los afectados, lo cual hizo que su confianza se debilitara.

3. Competencia. Esta se dio sobre la legitimidad de los resultados de los análisis de lixiviados; el Estado y Hasar's argumentaron que sus lixiviados no contaminan, mientras que los resultados de las comunidades arrojaron datos completamente diferentes.

4. Tensión en aumento. La incredulidad ante la propuesta del Estado de solucionar el problema de lixiviados ocasionó que las comunidades bloquearan el acceso a los sitios de disposición final y llamaran a los medios para que difundieran su caso, pero este no tuvo mucha cobertura.

5. Conflicto como tal. Las comunidades se han aliado con otros actores, han presentado el caso ante la CEDHJ y continúan luchando por una mejor calidad de vida.

6. Crisis. No ha habido manifestaciones violentas que terminen con la vida de alguno de los actores involucrados, pero las tensiones y el conflicto continúan.

\section{Conclusiones}

De lo anterior se puede inferir que el conflicto socioambiental provocado por los vertederos actuales y anteriores en el municipio de Zapopan, Jalisco, sigue latente. Aunque las comunidades de las localidades de Huaxtla, Milpillas y San Lorenzo han presentado su caso ante la CEDHJ, no han tenido una resolución favorable por parte del Estado; la contaminación del agua y el suelo con lixiviados está ocasionando la pérdida del ecosistema, con lo que se alteran la cadena trófica y los flujos de energía, lo cual afecta la calidad de vida de la población y altera su salud; estas son las causas que subyacen al conflicto socioambiental. Es importante resaltar que no se han realizado estudios epidemiológicos con los habitantes de la zona, pero de acuerdo con sus testimonios han presentado en forma recurrente enfermedades gastrointestinales y epidérmicas. 
Los actores protagonistas de este conflicto no son únicamente los de la comunidad afectada, el Estado y la empresa privada, por lo que se debe involucrar también a la población en general. Solo así se podrá contribuir a la protección del medio ambiente.

En resumen, con base en lo expuesto anteriormente, se concluye que los principios de este conflicto socioambiental surgen desde el momento en que se abrieron los primeros sitios de disposición final y la contaminación se hizo presente. La falta de respuesta del Estado ante la contaminación de la zona ha generado inconformidad, resistencia y lucha por parte de las comunidades. Las multas a los sitios de disposición no impactan su operación.

En el caso presentado está ocurriendo un proceso de lucha en que los actores sociales, en este caso los afectados, han construido redes de colaboración para hacer frente al problema. Inician con demandas, propuestas y estrategias para que el Estado y los agentes privados hagan una mejor gestión en materia ambiental.

Cabe apuntar que la población de los municipios de Zapopan y San Pedro Tlaquepaque aún no está tan involucrada en materia de RSU, ya que solo se actúa cuando se ve la afectación directa. Aunadas a esto, las invitaciones que hacen las instituciones a la población para que participe en la toma de decisiones en materia ambiental aún no permean en forma eficiente.

Casos como el expuesto demuestran el poder social, ya que los grupos organizados defienden su derecho a una mejor calidad de vida en un medio ambiente sano. Esto pone en evidencia la falta de aplicación de las políticas ambientales y su indiferencia ante la necesidad de resolver en forma positiva estos conflictos.

Es importante señalar que los conflictos socioambientales ponen en evidencia los efectos de la contaminación, el despojo y las luchas de poder entre los diferentes actores, mismos que contribuyen al deterioro ambiental.

\section{Referencias}

Ayuntamiento de Zapopan (2012). Estudio técnico justificativo para la declaratoria de Área Municipal de Protección Hidrológica Arroyo La Campana-Colomos III. Zapopan: Ayuntamiento de Zapopan. Recuperado de http://www.zapopan.gob. .mx/wp-content/uploads/2012/07/Protecci\%C3\%B3nHidrol\%C3\%B3gica.pdf

Bernache Pérez, G. (2011). Cuando la basura nos alcance. El impacto de la degradación ambiental. Guadalajara: CIESAS-Occidente, Universidad de Guadalajara.

Bernache Pérez, G. (2012). El confinamiento de la basura urbana y la contaminación de las fuentes de agua en México. Revista de El Colegio de San Luis, 1(1), 36-53. 
Buchanan, K. S. (2013). Contested discourses, knowledge, and socio-environmental conflict in Ecuador. Environmental Science \& Policy, 30, 19-25.

Cáceres, D. M. (2015). Accumulation by dispossession and socio-environmental conflicts caused by the expansion of agribusiness in Argentina. Journal of Agrarian Change, 15(1), 116-147.

Casillas, J. (s.f.). Pueblos de la barranca del río Santiago. Aproximación a su problemática y experiencia como afectados ambientales. Recuperado de http:// cronicadesociales.org/2010/05/17/pueblos-de-la-barranca-del-rio-santiago-en-jalisco/ (consultado el 1 de octubre de 2016).

Comisión Estatal de Derechos Humanos (CEDHJ) (2008). Informe 2007. Recupe- rado de https://cronicadesociales.org/2010/05/17/pueblos-de-la-barranca-del-riosantiago-enjalisco/ [consultado el 1 de octubre de 2016].

Comisión Estatal de Derechos Humanos (CEDHJ) (2011). Informe de actividades 20072012. Recuperado de http://cedhj.org.mx/informes/informe_2012.pdf [consultado el 30 de marzo de 2017].

Diario Oficial de la Federación (DOF) (2003). Ley General para la Prevención y Gestión Integral de los Residuos. Publicada el 8 de octubre de 2003. Última reforma publicada el 5 de diciembre de 2014.

Espluga, J., Paredes A., Gamer, N., \& Sola, R. (2008). El papel de la "confianza" en los conflictos socioambientales/The role of "trust" in socio-environmental conflicts. Política y Sociedad, 46(1), 255-277.

Fuenzalida, M. \& Quiroz, R. (2012). La dimensión espacial de los conflictos ambientales en Chile. Polis, 11(31), 157-168.

Galván Escobar, A. \& Huerta Macías, M. C. (2008). Análisis de las alteraciones geofísicas y riesgos naturales a consecuencia de la construcción de la presa Arcediano en la Barranca del Río Santiago zona Oblatos-Huentitán, zona metropolitana de Guadalajara. Sincronía, 4, 2.

Gómez, J. M. (2008). Los límites del consenso: la propuesta de desarrollo territorial rural en América Latina (pp. 249-274). En Fernandes, B. M. (org.), Campesinato e agronegócio na América Latina: a questao agrária atual. Buenos Aires: CLACSO.

Gran Castro, J. A. (2016). Los derechos humanos y la contaminación ambiental derivada de los sitios de disposición final de residuos en la ciudad de Guadalajara. Tesis de licenciatura en gestión y economía ambiental, Universidad de Guadalajara. Guadalajara, Jalisco.

Harvey, D. (2003). The new imperialism. Oxford: Oxford University Press.

Hilson, G. (2002). An overview of land use conflicts in mining communities. Land Use Policy, 19(1), 65-73.

Huérfano, V. (2010,17 de julio). Toman Picachos por 5 horas. Mural. Recuperado de 
https://cronicadesociales.org/2010/10/17/toman-picachos-por-5-horas/ [consultado el 13 de junio de 2016].

Instituto Nacional de Geografía y Estadística (INEGI) (2010a). Censo General de Población y Vivienda 2010. Recuperado de http://www.inegi.org.mx/est/ contenidos/ccv/ cpv2010/Default.aspx (consulado el 23 de septiembre de 2014).

Instituto Nacional de Geografía y Estadística (INEGI) (2010b). Cartografía geoestadística urbana 2010. Recuperado de http://www3.inegi,org.mx/sistemas/productos/ defaul.aspx? $\mathrm{c}=265 \&$ upc $=0 \& \mathrm{~s}=\mathrm{est} \& \mathrm{tg}=3604 \& \mathrm{f}=2 \& \mathrm{cl}=0 \& \mathrm{pf}=201100000 \& \mathrm{pg}=2$

Jalomo Aguirre, F. (2013). La metrópoli de Guadalajara y la política del agua: acciones pasadas y condiciones actuales. En Arellano Ríos, A. (coord.), Políticas y territorio. Una valoración de la acción gubernamental. Zapopan: El Colegio de Jalisco.

Leff, E. (1993). Cultura democrática, gestión ambiental y desarrollo sustentable en América Latina. Ecología Política, 4, 47-55.

Libiszewski, S. (1991). What is an environmental conflict? Journal of Peace Research, 28(4), 407-422.

Martín Beristain, C. (2007). El derecho a la reparación en los conflictos socioambientales. Bilbao: Universidad del País Vasco, Hegoa.

Martínez Alier, J. (1994). De la economía ecológica al ecologismo popular (2a ed.). Barcelona: Icaria Editorial.

Medina, M. (1999). Reciclaje de desechos sólidos en América Latina. Frontera Norte, 11(21), 1-25.

Merino Acuña, R. (2014). The politics of extractive governance: indigenous peoples and socio-environmental conflicts. The Extractive Industries and Society, 2(1), 85-92.

Montenegro Gómez, J. R. (2008). Los límites del consenso. La propuesta de desarrollo territorial rural en América Latina. Mançano Fernandes, B. Campesinato e agronegócio na América Latina: a questao agrária atual,(pp.249-274) Buenos Aires: CLACSO.

Orellana, R. (1999). Aproximaciones a un marco teórico para la comprensión y el manejo de conflictos socioambientales. En Ortiz, P. (comp.), Comunidades y conflictos socioambientales: experiencias y desafíos en América Latina. (pp. 89209) Quito: Ediciones UPS, Abya-Yala, Programa FTPP/FAO-Comunidec.

Organización Mundial de la Salud (OMS) (s.f.). El medio ambiente y la salud de los niños y sus madres. Recuperado de http://www.who.int/ceh/publications/factsheets/fs284/es/ [consultado el 13 de junio del 2016]

Pavón, D., Ventura, M., Ribas, A., Serra, P., Sauri, D., \& Breton, F. (2003). Land use change and socio-environmental conflict in the Alt Empordà county (Catalonia, Spain). Journal of Arid Environments, 54(3), 543-552. 
Pérez Serrano, G. (2002). Investigación cualitativa. Retos e interrogantes. Madrid: La Muralla.

Proceso (2010, 14 de febrero). Basurales asesinos. Recuperado de http://www. proceso.com.mx/82921 [consulado el 13 de junio de 2016]

Quintana Ramírez, A. P. (2008). El conflicto socioambiental y estrategias de manejo. Diplomado Plan de Ordenación y Manejo de la Cuenca del Río Salitre. Recuperado de http://pomcasalitre.files.wordpress.com/2010/02/modulo5conflicto-yresolucion. doc

Reboratti, C. (2012). Socio-environmental conflict in Argentina. Journal of Latin American Geography, 11(2), 3-20.

Rojas, D. F. \& Araujo, A. H. (2017). Desarrollo de una herramienta de vigilancia ambiental ciudadana basada en macroinvertebrados bentónicos en la cuenca del Jequetepeque (Cajamarca, Perú). Ecología Aplicada, 16(2), 105-114.

Sabatini, F., Mena, F., \& Vergara, P. (1996). El conflicto ambiental de Puchuncaví bajo democracia. Ambiente Hoy, 12(4), 30-40.

Secretaría de Medio Ambiente y Desarrollo Territorial, Subsecretaría de Fomento y Normatividad Ambiental (2004). NOM-083-SEMARNAT-2003 Especificaciones de protección ambiental para la selección del sitio, diseño, construcción, operación, monitoreo, clausura y obras complementarias de un sitio de disposición final de residuos sólidos urbanos y de manejo especial. Diario Oficial de la Federación. Recuperado de http://www.dof.gob.mx/nota_ detalle.php?codigo $=658648 \&$ fecha $=20 / 10 / 2004$ [consultado el 19 de octubre de 2014].

Tetreault, D. (2013). La megaminería en México. Reformas estructurales y resistencia. Letras Verdes, 14, 214-234.

Tetreault, D., Ochoa García, H., \& Hernández González, E. (2012a). Introducción. En Tetreault, D., Ochoa, H., \& Hernández, E. (comps.), Conflictos socioambientales y alternativas de la sociedad civil (pp. 13-26). Guadalajara: ITESO.

Tetreault, D., Ochoa García, H., Castillo Castro, X. C., Figueroa-Bautista, P., Guerritsen, P., Lezama-Escalante, C. ... \& Regalado-Santillán, J. (2012b). Conflictos socioambientales y alternativas de la sociedad civil. Guadalajara: ITESO.

Tetreault, D. y McCulligh, C. (2012). Panorama de conflictos socioambientales en Jalisco. En Tetreault, D., Ochoa, H., \& Hernández, E. (comps.), Conflictos socioambientales y alternativas de la sociedad civil (pp. 93-126). Guadalajara: ITESO.

Toledo, V. M., Garrido, D., \& Barrera Basols, N. (2013). Conflictos socioambientales, resistencias ciudadanas y violencia neoliberal en México. Ecología Política, 46, $115-124$.

Vargas, M. A, (2016). Vecinos bloquean Picachos. El Informador. Recuperado de http: 
//www.informador.com.mx/jalisco/2009/145419/6/vecinos-bloquean-picachos. htm [consultado el 13 de junio de 2016].

Velázquez López, L., Ochoa García, H., \& Morales Hernández, J. (2012). Agua y conflictos ambientales en la ribera de Cajititlán, Jalisco. En Tetreault, D., Ochoa, H., \& Hernández, E. (comps.), Conflictos socioambientales y alternativas de la sociedad civil, (pp. 181-214). Guadalajara: ITESO.

Verduzco Chávez, B. (2002). Conflictos ambientales. La internacionalización de la defensa de las comunidades contra instalaciones contaminantes. Guadalajara: Universidad de Guadalajara.

Walter, M. (2009). Conflictos ambientales, socioambientales, ecológico distributivos, de contenido ambiental... Reflexionando sobre enfoques y definiciones. Boletín Ecos, 6, 1-9. 